\title{
Clarithromycin resistance and female gender affect Helicobacter pylori eradication failure in chronic gastritis
}

\author{
Young Woon Chang ${ }^{1,}$, Weon Jin $\mathrm{Ko}^{2,{ }^{*}}$, Chi Hyuk Oh${ }^{1}$, Yoo Min Park ${ }^{1}$, Shin Ju Oh${ }^{1}$, Jung Rock Moon ${ }^{1}$, \\ Jun-Hyung $\mathrm{Cho}^{3}$, Jung-Wook $\mathrm{Kim}^{1}$, and Jae-Young Jang ${ }^{1}$
}

${ }^{1}$ Department of Internal Medicine, Kyung Hee University School of Medicine, Seoul; ${ }^{2}$ Department of Internal Medicine, CHA Gumi Medical Center, CHA University School of Medicine, Gumi; ${ }^{3}$ Department of Internal Medicine, Soonchunhyang University College of Medicine, Seoul, Korea
Received: February 8, 2018 Revised : March 8, 2018 Accepted: March 9, 2018

\section{Correspondence to}

Chi Hyuk Oh, M.D.

Division of Gastroenterology and Hepatology, Department of Internal medicine, Kyung Hee University School of Medicine, 23 Kyungheedae-ro, Dongdaemun-gu, Seoul 02447, Korea

Tel: $+82-2-958-8114$

Fax: +82-2-958-8147

E-mail: harrison@daum.net

*These authors contributed equally to this work.
Background/Aims: The eradication rate of the first-line triple therapy (a proton pump inhibitor, clarithromycin, and amoxicillin) for Helicobacter pylori infection has gradually decreased in Korea. We evaluated whether clinical parameters, clarithromycin resistance, and $\mathrm{CrP}_{2} \mathrm{C}_{1}$ genotype can affect the eradication failure.

Methods: A total of 203 patients with $\mathrm{H}$. pylori-positive chronic gastritis were consecutively enrolled. They received clarithromycin-based triple therapy for 7 days. A clarithromycin resistance test was performed by detection of $\mathrm{A} 2142 \mathrm{G}$ and A2143G point mutations in $\mathrm{H}$. pylori $23 \mathrm{~S}$ rRNA. The $\mathrm{CrP}_{2} \mathrm{C}_{19}$ genotype was examined for polymorphism G681A of exon 5 and G636A of exon 4 by polymerase chain reaction with restriction fragment length polymorphism. Eradication was assessed by a ${ }^{13} \mathrm{C}$-urea breath test 4 weeks after treatment.

Results: Of 203 patients, 190 completed the study. The eradication rate was $64.0 \%$ according to intention-to-treat analysis and $68.4 \%$ by per-protocol analysis. $\mathrm{Cr}$ $\mathrm{P}_{2} \mathrm{C}_{1} 9$ genotypes were identified as follows: 75 poor metabolizers, 75 intermediate metabolizers, and 40 rapid metabolizers. Nonetheless, this polymorphism was not significantly associated with eradication failure $(p=0.682)$. Clarithromycin resistance was detected in 33/190 patients (17.4\%), and their eradication rate was zero. Clarithromycin resistance (odds ratio [OR], 19.13; 95\% confidence interval [CI], 9.35 to 35.09) and female gender (OR, 1.73; 95\% CI, 1.15 to 4.25) were significantly associated with eradication failure. The other clinical parameters such as age, cigarette smoking, alcohol intake, the body mass index, hypertension, and diabetes were not significantly associated with eradication.

Conclusions: Clarithromycin resistance and female gender are factors affecting $H$. pylori eradication failure in patients with chronic gastritis.

Keywords: Helicobacter pylori; Eradication; Clarithromycin; Resistance; CYP2C19 genotype

\section{INTRODUCTION}

Helicobacter pylori infection affects $54.4 \%$ of population in Korea [1]. H. pylori infection can cause chronic gastri- tis, peptic ulcer disease (PUD), mucosa-associated lymphoid tissue lymphoma, and gastric cancer $[2,3]$. Currently, most guidelines recommend a triple therapy consisting of a proton pump inhibitor (PPI) and two 
antibiotics (clarithromycin [CAM] and amoxicillin) as the first-line treatment for $H$. pylori eradication.

Nonetheless, the eradication rate of this regimen has decreased to an unacceptable level in Korea. It means that the eradication rate is below $80 \%$ according to intention-to-treat (ITT) analysis and below $90 \%$ by the per-protocol (PP) analysis $[4,5]$. Despite the disappointing results, the Korean medical insurance system still considers the CAM-based triple therapy as the first-line treatment. A lack of sufficient evidence is the reason why the CAM-based regimen is not changed to other treatment regimens.

The eradication failure is influenced by host-related, bacterial, and environmental factors $[6,7]$. CAM resistance is considered the most important factor of eradication failure [8]. The CAM resistance rate is constantly increasing worldwide and varies substantially by region $[9,10]$. It has been reported that the CAM resistance rate gradually increased from $17.2 \%$ to $23.7 \%$ between 2003 and 2012 in Korea [11]. As a host factor, the $\mathrm{CYP}_{2} \mathrm{C}_{19}$ polymorphism is also related to the $H$. pylori eradication rate because cytochrome $\mathrm{P}_{45}{ }_{2} \mathrm{C}_{19}\left(\mathrm{CYP}_{2} \mathrm{C}_{19}\right)$ is involved in PPI metabolism [12]. The rapid metabolizer (RM) genotype of $\mathrm{CYP}_{2} \mathrm{C}_{1} 9$ can be unfavorable for eradication because of low bioavailability of PPI. The roles of other factors including age, gender, the body mass index (BMI), cigarette smoking, alcohol intake, and systemic diseases are still controversial [5,13,14].

The aim of the present study was to determine whether CAM resistance, the $\mathrm{CYP}_{2} \mathrm{C}_{19}$ genotype, and clinical parameters can affect $H$. pylori eradication in a Korean population.

\section{METHODS}

\section{Patients and study protocol}

We consecutively enrolled a total of $203 \mathrm{H}$. pylori-positive patients with chronic gastritis from September 2014 to August 2015 at Kyung Hee University Hospital, Seoul, Korea. The participants were excluded from the study if they had a PUD, gastric cancer, previous gastrectomy, severe underlying diseases, or received antibiotics or PPI within 4 weeks [15]. At the first time of enrollment, we tried to include patients with PUD and chronic gastritis, but the number of $H$. pylori-related
PUD cases was much smaller relative to chronic gastritis. Therefore, we included only chronic atrophic or nonatrophic gastritis. All the participants received a diagnosis of $H$. pylori infection by a rapid urease test or detection of $H$. pylori genomic DNA from gastric mucosal tissues.

We collected data including age, gender, cigarette smoking, alcohol intake, salt in the diet, family history of gastric cancer, BMI, hypertension, and diabetes. Although we tried to acquire prior history of antibiotic use, most subjects did not remember the name or the amount used of antibiotics. All the patients were prescribed the triple regimen consisting of a standard dose of PPI (pantoprazole), $1 \mathrm{~g}$ of amoxicillin, and $0.5 \mathrm{~g}$ of CAM twice daily for 7 days. Four weeks after the end of eradication therapy, the ${ }^{13} \mathrm{C}$-urea breath test was performed to confirm successful eradication of $H$. pylori infection. A week after completion of treatment, information about adverse events and compliance was collected from the outpatient department by means of telephone interviews. When patients had consumed more than $85 \%$ of the study medications, compliance was considered satisfactory. The adverse events were metallic taste, diarrhea, nausea, epigastric discomfort, urticaria, and headache. All the patients provided written informed consent prior to enrollment. The Institutional Review Board of the Kyung Hee University Hospital approved the study protocol (KMC IRB 1427-01).

\section{CAM resistance test}

It is known that the mutation of $23 \mathrm{~S}$ rRNA results in increased antibiotic resistance [16]. CAM resistance of $H$. pylori was determined by analysis of the $\mathrm{A} 2142 \mathrm{G}$ or A2143G mutation in $23 \mathrm{~S}$ rRNA. $H$. pylori genomic DNA was extracted from two biopsy specimens of the antrum and body collected during endoscopy. DNA was amplified by Seeplex Home-brew primer mix of the Seeplex ClaR-H. pylori polymerase chain reaction (PCR) kit (Seegene Inc., Seoul, Korea) that was developed using the dual-priming oligonucleotide system to detect the A2142G or A2143G mutation.

\section{$\mathrm{CrP}_{2} \mathrm{C}_{19}$ genotyping}

The genotyping of $\mathrm{CYP}_{2} \mathrm{C}_{19}$ was conducted using PCR with restriction fragment length polymorphism [17]. To detect the polymorphism of $\mathrm{CrP}_{2} \mathrm{C}_{19}$ in exon 5, we de- 


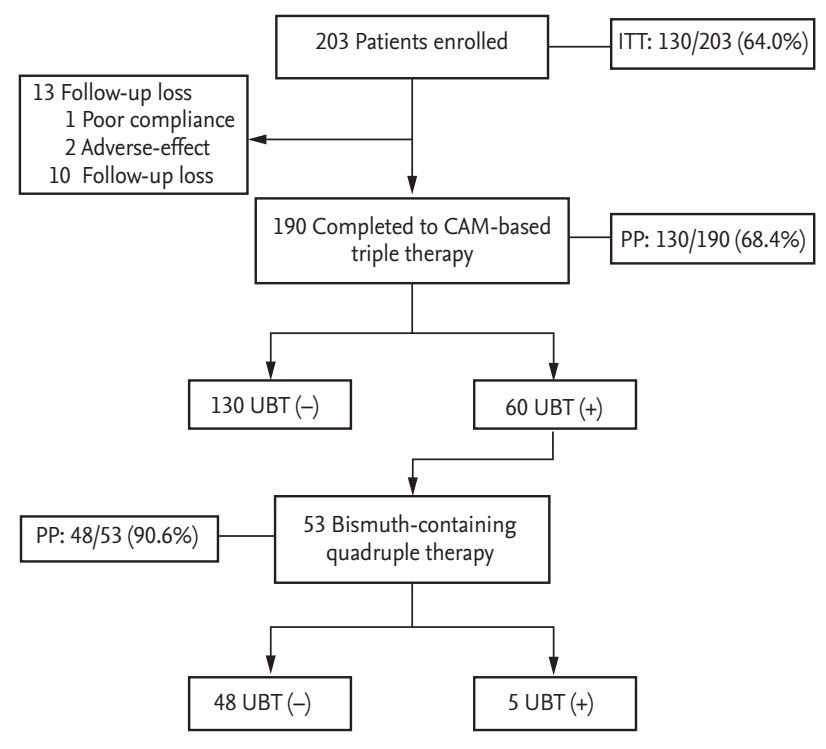

Figure 1. The flow chart of enrollment in this study. ITT, intention-to-treat; CAM, clarithromycin; UBT, urea breath test; PP, per-protocol.

signed the sense (5'-AATTACAACCAGAGCT'TGGC-3') and antisense primers ( 5 '-TATCACTTTCCATAAAAGCAAG- $\left.3^{\prime}\right)$. In exon 4 , we used the sense (5'-GCTTCACCCTGTGATCCCAC- $\left.3^{\prime}\right)$ and antisense primer (5'-TCAGGGCTTGGTCAATATAG-3'). The PCR product containing G681A in exon 5 was digested by incubation with $\mathrm{Sma} \mathrm{I}$ at $37^{\circ} \mathrm{C}$ for 3 hours, resulting in two bands (120 and $49 \mathrm{bp}$ ) for the G/G genotype (wild), three bands $(169,120$, and $49 \mathrm{bp})$ for the G/A genotype (heterozygote), and one band of $169 \mathrm{bp}$ for the A/A genotype (mutant type). The PCR product containing G636A in exon 4 was digested by incubation with $\mathrm{BamH} \mathrm{I}$ at $37^{\circ} \mathrm{C}$ for 3 hours, resulting in two bands (155 and $93 \mathrm{bp}$ ) for the G/ G genotype (wild type), three bands $(248,155$, and $93 \mathrm{bp}$ ) for the G/A genotype (heterozygote), and one band of $248 \mathrm{bp}$ for the A/A genotype (mutant type). The patients were classified into three groups by $\mathrm{CrP}_{2} \mathrm{C}_{19}$ genotype: RM had exon 5, wild type/exon 4, wild type; intermediate metabolizers (IMs) had exon 5, wild type/exon 4, heterozygote or exon 5, heterozygote/exon 4, wild type; and poor metabolizers (PMs) had both mutated alleles.

\section{Data analysis}

Eradication was evaluated by the ITT and PP analyses with $95 \%$ confidence intervals (CIs). Continuous vari- ables are expressed as mean \pm standard deviation, and categorical variables as percentages. Student $t$ test for parametric data and the Mann-Whitney $U$ test for nonparametric data were used for comparisons of the means of continuous variables, whereas differences in categorical variables were assessed using chi-square or Fisher exact test for demographic and clinical data. Logistic regression analyses were conducted to determine the independent contribution of each possible predictor to eradication failure. PASW Statistics for Windows version 22.0 (IBM Co., Armonk, NY, USA) was used for statistical analysis. All statistical tests were two-tailed, and $p$ values $<0.05$ were assumed to indicate statistical significance.

\section{RESULTS}

\section{Baseline characteristics of the study population}

Thirteen cases among the total of 203 patients did not participate. After receiving CAM-based triple therapy, 10 cases were lost to follow-up, one case was non-compliant, and two cases discontinued treatment owing to severe adverse events. Eventually, 190 patients were analyzed (Fig. 1). The baseline characteristics of all the participants are summarized in Table 1.

\section{Eradication rates and adverse events}

The overall eradication rates of CAM-based standard triple therapy according to ITT and PP analyses were 64.0\% (130/203 patients) and 68.4\% (130/190 patients), respectively (Table 1$)$. The frequency of adverse events is shown in Table 2. The most frequent adverse event was metallic taste (65/190, 34.2\%). Diarrhea, nausea, epigastric discomfort, urticaria, and headache followed. The adverse events were not different between the success group and failure group. Among 60 patients with first eradication failure, 53 patients received 10 days of bismuth-containing quadruple therapy. In 48 patients, the $H$. pylori infection was eradicated with a quadruple therapy $(48 / 53,90.6 \%)$ (Fig. 1).

\section{CAM resistance}

The rate of CAM resistance was 17.4\% (33/190 cases) (Table 1). The A2143G mutation was predominantly observed (30/33 cases, 90.9\%). Two cases (6.1\%) had A2142G 
Table 1. Demographic and clinical characteristics of all the patients $(n=190)$

\begin{tabular}{|c|c|}
\hline Variable & Value \\
\hline Age, yr & $55.4 \pm 10.2$ \\
\hline Male:Female ratio & $89: 101(46.8: 53.2)$ \\
\hline Cigarette smoking & $31(16.3)$ \\
\hline Alcohol intake & $65(34.2)$ \\
\hline High salt diet & $74(38.9)$ \\
\hline Family history of GC & $23(12.1)$ \\
\hline \multicolumn{2}{|l|}{ Underlying disease } \\
\hline Hypertension & $49(25.8)$ \\
\hline Diabetes & $15(7.9)$ \\
\hline Body mass index, $\mathrm{kg} / \mathrm{m}^{2}$ & $23.8 \pm 3.0$ \\
\hline \multicolumn{2}{|l|}{ Endoscopic findings } \\
\hline Non-atrophic gastritis & $71(37 \cdot 4)$ \\
\hline Atrophic gastritis & $119(62.6)$ \\
\hline CAM mutation & $33(17 \cdot 4)$ \\
\hline A2142G only & $2(6.1)$ \\
\hline A2143G only & $30(90.9)$ \\
\hline$A 2142 \mathrm{G}+\mathrm{A} 2143 \mathrm{G}$ & $1(3.0)$ \\
\hline \multicolumn{2}{|l|}{$\mathrm{CrP}_{2} \mathrm{C}_{19}$ genotype } \\
\hline $\mathrm{PM}$ & $74(38.9)$ \\
\hline $\mathrm{IM}$ & $75(39 \cdot 5)$ \\
\hline $\mathrm{RM}$ & $41(21.6)$ \\
\hline \multicolumn{2}{|l|}{ Eradication rate } \\
\hline IT'T analysis & $130 / 203(64.0)$ \\
\hline PP analysis & $130 / 190(68.4)$ \\
\hline
\end{tabular}

Values are presented as mean \pm SD or number (\%).

GC, gastric cancer; CAM, clarithromycin; PM, poor metabolizer; IM, intermediate metabolizer; RM, rapid metabolizer; ITT, intention-to-treat; PP, per-protocol.

mutation, and one case had a dual point mutation at A2142G and A2143G. All patients with CAM resistance revealed eradication failure (Table 3). The frequency of CAM resistance was different between females (20/total 101 patients, $19.8 \%$ ) and males (13/total 89 patients, 14.6\%), but the difference did not reach statistical significance $(p=0.087)$.

\section{$\mathrm{CrP}_{2} \mathrm{C}_{19}$ genotype}

Analysis of the $\mathrm{CrP}_{2} \mathrm{C}_{1} 9$ genotypes showed that the RM genotype was present in 40/190 cases (21.0\%), IM genotype in 75/190 cases (39.5\%), and PM genotype in 75/190
Table 2. The frequency of adverse events in eradication success and failure groups

\begin{tabular}{lccc}
\hline Variable & $\begin{array}{c}\text { Success } \\
\text { group } \\
(\mathrm{n}=130)\end{array}$ & $\begin{array}{c}\text { Failure group } \\
(\mathrm{n}=60)\end{array}$ & p value \\
\hline None & $48(36.9)$ & $17(28.3)$ & 0.324 \\
Metallic taste & $67(51.5)$ & $34(56.7)$ & 0.535 \\
\hline Diarrhea & $18(13.8)$ & $8(13.3)$ & 1.000 \\
Nausea & $5(3.8)$ & $1(1.7)$ & 0.667 \\
$\begin{array}{l}\text { Epigastric } \\
\text { discomfort }\end{array}$ & $7(5.4)$ & $6(10.0)$ & 0.353 \\
Urticaria & $3(2.3)$ & $2(3.3)$ & 0.652 \\
\hline $\begin{array}{l}\text { Headache/ } \\
\text { dizziness }\end{array}$ & $8(6.2)$ & $7(11.7)$ & 0.247 \\
\hline
\end{tabular}

Values are presented as number (\%).

cases (39.5\%). There were no statistically significant differences among the three genotypes in $\mathrm{H}$. pylori eradication $(p=0.682)$ (Table 3$)$.

\section{Factors affecting eradication failure}

The clinical characteristics of the eradication success group and failure group are shown in Table 3. In univariate analysis, there were no statistically significant differences in clinical parameters except for female gender. CAM resistance was detected in 33/190 (17.4\%) of cases and their eradication rate was zero (o/33 cases). In multivariate logistic regression analysis, female gender (odds ratio [OR], 1.73; 95\% CI, 1.15 to $4.251 ; p=0.016$ ) and CAM resistance (OR, 19.13; 95\% CI, 9.354 to 35.094; $p$ $<0.001$ ) were identified as powerful predictive factors of eradication failure (Table 4).

\section{DISCUSSION}

The rate of $H$. pylori eradication by the CAM-based triple regimen has been decreasing in the past decades in Korea [18]. In this study, we aimed to analyze the several factors associated with eradication failure; especially we focused on CAM resistance and the $\mathrm{CrP}_{2} \mathrm{C}_{1} 9$ genotype. Interestingly, in our study, all patients with CAM resistance showed eradication failure. In several previous studies, patients treated with the CAM-based triple regimen showed an approximately $20 \%$ to $22 \%$ eradication 
Table 3. Clinical characteristics in eradication success and failure groups

\begin{tabular}{|c|c|c|c|}
\hline Variable & $\begin{array}{l}\text { Success } \\
\text { group } \\
(\mathrm{n}=130)\end{array}$ & $\begin{array}{l}\text { Failure group } \\
\qquad(\mathrm{n}=60)\end{array}$ & $p$ value \\
\hline Age, yr & $55 \cdot 3 \pm 10.3$ & $55 \cdot 5 \pm 10.1$ & 0.911 \\
\hline Male:Female ratio & $\begin{array}{c}68: 62 \\
(52.3: 47 \cdot 7)\end{array}$ & $\begin{array}{c}21: 39 \\
(35.0: 65.0)\end{array}$ & 0.039 \\
\hline Cigarette smoking & $24(18.5)$ & $7(11.7)$ & 0.334 \\
\hline Alcohol intake & $50(38.5)$ & $15(25.0)$ & 0.098 \\
\hline High salt diet & $49(37.7)$ & $25(41.7)$ & 0.717 \\
\hline $\begin{array}{l}\text { Family history } \\
\text { of GC }\end{array}$ & $14(10.8)$ & $9(15.0)$ & 0.554 \\
\hline \multicolumn{4}{|l|}{ Underlying disease } \\
\hline Hypertension & $36(27.7)$ & $13(21.7)$ & 0.481 \\
\hline Diabetes & $13(10.8)$ & $1(1.7)$ & 0.061 \\
\hline $\mathrm{BMI}, \mathrm{kg} / \mathrm{m}^{2}$ & $23.8 \pm 2.9$ & $23.9 \pm 3.2$ & 0.902 \\
\hline Endoscopic findings & & & 0.728 \\
\hline $\begin{array}{l}\text { Non-atrophic } \\
\text { gastritis }\end{array}$ & $47(36.2)$ & $24(40.0)$ & \\
\hline Atrophic gastritis & $83(63.8)$ & $36(60.0)$ & \\
\hline CAM mutation & o & $33(55 \cdot 0)$ & $<0.001$ \\
\hline $\mathrm{CYP}_{2} \mathrm{C}_{19}$ genotype & & & 0.733 \\
\hline $\mathrm{PM}$ & $52(40.0)$ & $22(36.7)$ & \\
\hline IM & $52(40.0)$ & $23(38.8)$ & \\
\hline $\mathrm{RM}$ & $26(20.0)$ & $15(21.6)$ & \\
\hline
\end{tabular}

Values are presented as mean $\pm \mathrm{SD}$ or number (\%). GC, gastric cancer; BMI, body mass index; CAM, clarithromycin; PM, poor metabolizer; IM, intermediate metabolizer; RM; rapid metabolizer.

rate $[19,20]$. In those studies, resistance to CAM was evaluated using the agar dilution method. In contrast, our study used dual-priming oligonucleotide PCR to identify the point mutation, with higher specificity for detection of CAM resistance.

The Maastricht V/Florence Consensus Report in 2016 stated that PPI-CAM-amoxicillin triple therapy without a prior susceptibility test should be reconsidered in a region where the CAM resistance rate is higher than $15 \%$ [15]. In our study, the CAM resistance was $17.4 \%$. In another recent Korean study, the CAM resistance rate was above 20\% [14]. Considering that the CAM resistance rate was over $15 \%$, alternative regimens are needed as the first-line treatment in Korea.
Table 4. Logistic regression analysis for Helicobacter pylori eradication failure

\begin{tabular}{lccr}
\hline Variable & Adjusted OR & $95 \%$ CI & p value \\
\hline Female sex & 1.730 & $1.151-4.251$ & 0.016 \\
CAM resistance & 19.132 & $9.354-35.094$ & $<0.001$ \\
\hline
\end{tabular}

OR, odds ratio; CI, confidence interval; CAM, clarithromycin.

CAM resistance is related to $\mathrm{A} 2142 \mathrm{G}, \mathrm{A} 2143 \mathrm{G}$, or A2142C point mutations in the $23 \mathrm{~S}$ rRNA of $H$. pylori, which decrease the affinity of CAM binding to the ribosome. Among the three point mutations, the A2143G point mutation is the major cause of resistance [21,22]. In our study, A2143G rather than $\mathrm{A} 2142 \mathrm{G}$ was also found to be the most prevalent mutation attributed to CAM resistance.

We examined a $\mathrm{CrP}_{2} \mathrm{C}_{19}$ polymorphism to find the effect of PPI pharmacokinetics on $\mathrm{H}$. pylori eradication. Depending on the $\mathrm{CrP}_{2} \mathrm{C}_{1} 9$ genotype, the effect of PPI prescription on the eradication rate can vary. By contrast to the first-generation PPIs (e.g., omeprazole, pantoprazole, and lansoprazole), the second-generation PPIs (e.g., esomeprazole and rabeprazole) are not affected by $\mathrm{CrP}_{2} \mathrm{C}_{19}$ metabolism [23-25]. There are a few studies on $\mathrm{CYP}_{2} \mathrm{C}_{19}$ genetic variations and the eradication rate in Korea. One study revealed that the eradication rate of an esomeprazole-based regimen is higher (88.3\%) than that of a pantoprazole-based regimen (82.6\%) after adjustment for the $\mathrm{CYP}_{2} \mathrm{C}_{1} 9$ genotype. Nonetheless, the differences were not statistically significant [26]. In our study, all the patients were treated with pantoprazole because we believe that pantoprazole has higher antacid secretory efficacy than omeprazole does [27]. Consequently, the eradication rate was not significantly influenced by the $\mathrm{CrP}_{2} \mathrm{C}_{19}$ genotype. Nevertheless, it is possible that if we had treated the patients with omeprazole instead of pantoprazole, the results might have been different.

There are several reports that female gender can influence $H$. pylori eradication $[28,29]$. One study suggested that there might be a difference in gastric physiology between males and females [30]. Another study indicates that females are preferentially infected with the $H$. pylori strain with $\mathrm{A} 2143 \mathrm{G}$ mutation, and thus the point mutation can contribute to the eradication failure [31]. 
Our study showed that the female gender is an unfavorable factor affecting eradication. We found that females had a higher CAM resistance rate compared with males, but the difference was not significant $(p=0.087)$. The cause of gender differences in the eradication rate needs further research.

Cigarette smoking has been reported as a contributing factor of $\mathrm{H}$. pylori eradication failure because of decreased blood flow to the gastric mucosa and stimulation of acid secretion. Furthermore, cigarette smoking provokes acid secretion and thus reduces the activity of acid-sensitive antibiotics (e.g., amoxicillin) [30]. In the present study, however, we did not find an association between current cigarette smoking and eradication failure.

It has been reported that age can affect the eradication rate. Elderly patients with atrophic gastritis followed by decreased gastric acid secretion show a higher eradication rate $[32,33]$. In the present study, age was not significantly associated with eradication outcomes.

Several studies have assessed the possible association between $\mathrm{H}$. pylori and alcohol intake. Of note, one study showed that alcoholic beverages have some bactericidal activity. For this reason, regular wine (1 glass/day) or beer (0.5 pint/day) consumption was found to prevent $H$. pylori infection [34]. In addition, some studies have indicated that alcohol consumption contributes to the efficacy of $H$. pylori eradication [35,36]. Nonetheless, self-reported alcohol consumption is not accurate. This inaccuracy is a possible limitation of our study and studies by others. We could not find any association between alcohol intake and eradication efficacy.

Underlying chronic diseases such as hypertension and diabetes were occasionally reported to contribute to eradication failure, but the results were inconsistent, and the evidence was limited [37,38].

Our study has some limitations. First, we included only the group of chronic gastritis, but not other gastroduodenal-disease groups. A further study including various gastroduodenal diseases is needed in the future. Second, we used only a CAM resistance test by means of a dual-priming oligonucleotide system. We did not perform amoxicillin resistance test. The resistance rate of amoxicillin in Korea has increased from $7.4 \%$ in 2008 to $17.2 \%$ in 2013 [11]. Nevertheless, amoxicillin resistance had little or no effect on $\mathrm{H}$. pylori eradication. Furthermore, the eradication rate was found to be $100 \%$ in a
CAM-sensitive and amoxicillin-resistant $H$. pylori strain in a Korean population [8,39]. In addition, a recent Korean study revealed that eradication failure was not significantly affected by amoxicillin resistance [14]. Considering the other investigators' results, we assumed that doing only the CAM resistance test without an amoxicillin resistance test would not affect our results strongly.

Third, we treated the patients with one type of PPIs, pantoprazole. Our result is not sufficient to confirm irrelevance of the $\mathrm{CrP}_{2} \mathrm{C}_{1}$ genotype for eradication failure and because pantoprazole is less affected by CY$\mathrm{P} 2 \mathrm{C} 19$ metabolic pathway than omeprazole is.

In conclusion, CAM resistance and female gender can affect the failure of $\mathrm{H}$. pylori eradication with the CAMbased standard triple therapy in patients with chronic gastritis. A CAM resistance test before treatment is needed for improvement of the eradication rate and for reducing the overuse of antibiotics. Additionally, we propose that the CAM-based triple therapy should be replaced by some other first-line treatment not including CAM in high CAM resistance countries such as Korea.

\section{KEY MESSAGE}

1. The eradication rate of a clarithromycin-based triple regimen has decreased to an unacceptable level in Korea. We found that clarithromycin resistance is the most powerful predictive factor of Helicobacter pylori eradication failure in Korea.

2. To increase the rate of successful eradication, new treatment guidelines and an appropriate treatment strategy are necessary.

\section{Conflict of interest}

No potential conflict of interest relevant to this article was reported.

\section{Acknowledgments}

We appreciate the excellent support of our nursing team at the Kyung Hee Medical Center (KHMC) Digestive Endoscopy Center. 


\section{REFERENCES}

1. Lim SH, Kwon JW, Kim N, et al. Prevalence and risk factors of Helicobacter pylori infection in Korea: nationwide multicenter study over 13 years. BMC Gastroenterol 2013;13:104.

2. Franchini M, Cruciani M, Mengoli C, Pizzolo G, Veneri D. Effect of Helicobacter pylori eradication on platelet count in idiopathic thrombocytopenic purpura: a systematic review and meta-analysis. J Antimicrob Chemother 2007;60:237-246.

3. McColl KE. Clinical practice. Helicobacter pylori infection. N Engl J Med 2010;362:1597-1604.

4. Cho DK, Park SY, Kee WJ, et al. The trend of eradication rate of Helicobacter pylori infection and clinical factors that affect the eradication of first-line therapy. Korean J Gastroenterol 2010;55:368-375.

5. Malfertheiner P, Chan FK, McColl KE. Peptic ulcer disease. Lancet 2009;374:1449-1461.

6. Sugimoto M, Uotani T, Sahara S, et al. Efficacy of tailored Helicobacter pylori eradication treatment based on clarithromycin susceptibility and maintenance of acid secretion. Helicobacter 2014;19:312-318.

7. Kim SE, Park MI, Park SJ, et al. Trends in Helicobacter pylori eradication rates by first-line triple therapy and related factors in eradication therapy. Korean J Intern Med 2015;30:801-807.

8. Hwang TJ, Kim N, Kim HB, et al. Change in antibiotic resistance of Helicobacter pylori strains and the effect of A2143 G point mutation of $23 \mathrm{~S}$ rRNA on the eradication of H. pylori in a single center of Korea. J Clin Gastroenterol 2010;44:536-543.

9. Mitchell H, Katelaris P. Epidemiology, clinical impacts and current clinical management of Helicobacter pylori infection. Med J Aust 2016;204:376-380.

10. Megraud F. Helicobacter pylori and antibiotic resistance. Gut 2007;56:1502.

11. Lee JW, Kim N, Kim JM, et al. Prevalence of primary and secondary antimicrobial resistance of Helicobacter pylori in Korea from 2003 through 2012. Helicobacter 2013;18:206-214.

12. Dickson EJ, Stuart RC. Genetics of response to proton pump inhibitor therapy: clinical implications. Am J Pharmacogenomics 2003;3:303-315.

13. Suzuki T, Matsuo K, Ito H, et al. Smoking increases the treatment failure for Helicobacter pylori eradication. Am
J Med 2006;119:217-224.

14. Lee JY, Kim N, Kim MS, et al. Factors affecting first-line triple therapy of Helicobacter pylori including CY$\mathrm{P}_{2} \mathrm{C}_{1} 9$ genotype and antibiotic resistance. Dig Dis Sci 2014;59:1235-1243.

15. Malfertheiner P, Megraud F, O'Morain CA, et al. Management of Helicobacter pylori infection-the Maastricht V/ Florence Consensus Report. Gut 2017;66:6-30.

16. Occhialini A, Urdaci M, Doucet-Populaire F, Bebear CM, Lamouliatte H, Megraud F. Macrolide resistance in Helicobacter pylori: rapid detection of point mutations and assays of macrolide binding to ribosomes. Antimicrob Agents Chemother 1997;41:2724-2728.

17. Andersson T, Regardh CG, Lou YC, Zhang Y, Dahl ML, Bertilsson L. Polymorphic hydroxylation of S-mephenytoin and omeprazole metabolism in Caucasian and Chinese subjects. Pharmacogenetics 1992;2:25-31.

18. Yoon JH, Baik GH, Sohn KM, et al. Trends in the eradication rates of Helicobacter pylori infection for eleven years. World J Gastroenterol 2012;18:6628-6634.

19. Park CS, Lee SM, Park CH, et al. Pretreatment antimicrobial susceptibility-guided vs. clarithromycin-based triple therapy for Helicobacter pylori eradication in a region with high rates of multiple drug resistance. Am J Gastroenterol 2014;109:1595-1602.

20. Zheng Q, Chen WJ, Lu H, Sun QJ, Xiao SD. Comparison of the efficacy of triple versus quadruple therapy on the eradication of Helicobacter pylori and antibiotic resistance. J Dig Dis 2010;11:313-318.

21. De Francesco V, Margiotta M, Zullo A, et al. Prevalence of primary clarithromycin resistance in Helicobacter pylori strains over a 15 year period in Italy. J Antimicrob Chemother 2007;59:783-785.

22. Francavilla R, Lionetti E, Castellaneta S, et al. Clarithromycin-resistant genotypes and eradication of Helicobacter pylori. J Pediatr 2010;157:228-232.

23. Padol S, Yuan Y, Thabane M, Padol IT, Hunt RH. The effect of $\mathrm{CYP}_{2} \mathrm{C}_{19}$ polymorphisms on $\mathrm{H}$. pylori eradication rate in dual and triple first-line PPI therapies: a meta-analysis. Am J Gastroenterol 2006;101:1467-1475.

24. Tang HL, Li Y, Hu YF, Xie HG, Zhai SD. Effects of CY$\mathrm{P}_{2} \mathrm{C} 19$ loss-of-function variants on the eradication of $\mathrm{H}$. pylori infection in patients treated with proton pump inhibitor-based triple therapy regimens: a meta-analysis of randomized clinical trials. PLoS One 2013;8:e62162.

25. McNicholl AG, Linares PM, Nyssen OP, Calvet X, Gis- 
bert JP. Meta-analysis: esomeprazole or rabeprazole vs. first-generation pump inhibitors in the treatment of Helicobacter pylori infection. Aliment Pharmacol Ther 2012;36:414-425.

26. Kang JM, Kim N, Lee DH, et al. Effect of the CYP2C19 polymorphism on the eradication rate of Helicobacter pylori infection by 7-day triple therapy with regular proton pump inhibitor dosage. J Gastroenterol Hepatol 2008;23(8 Pt 1):1287-1291.

27. Pratha VS, Hogan DL, Lane JR, et al. Inhibition of pentagastrin-stimulated gastric acid secretion by pantoprazole and omeprazole in healthy adults. Dig Dis Sci 2006;51:123131.

28. Cai W, Zhou L, Ren W, Deng L, Yu M. Variables influencing outcome of Helicobacter pylori eradication therapy in South China. Helicobacter 2009;14:91-96.

29. Osato MS, Reddy R, Reddy SG, Penland RL, Malaty HM, Graham DY. Pattern of primary resistance of Helicobacter pylori to metronidazole or clarithromycin in the United States. Arch Intern Med 2001;161:1217-1220.

30. Moayyedi P, Chalmers DM, Axon AT. Patient factors that predict failure of omeprazole, clarithromycin, and tinidazole to eradicate Helicobacter pylori. J Gastroenterol 1997;32:24-27.

31. Kim T, Song HJ, Shin SY, et al. Clarithromycin-resistant Helicobacter pylori associated with $23 \mathrm{~S}$ rRNA point mutations in Jeju Island. Korean J Gastroenterol 2013;61:252258.

32. Treiber G, Ammon S, Klotz U. Age-dependent eradication of Helicobacter pylori with dual therapy. Aliment Phar- macol Ther 1997;11:711-718.

33. Sjostedt S, Sagar M, Lindberg G, Wikstrom B, Nord CE, Seensalu R. Prolonged and profound acid inhibition is crucial in Helicobacter pylori treatment with a proton pump inhibitor combined with amoxicillin. Scand J Gastroenterol 1998;33:39-43.

34. Murray LJ, Lane AJ, Harvey IM, Donovan JL, Nair P, Harvey $R$ F. Inverse relationship between alcohol consumption and active Helicobacter pylori infection: the Bristol Helicobacter project. Am J Gastroenterol 2002;97:27502755 .

35. Namiot Z, Namiot DB, Kemona A, Golebiewska M, Bucki $\mathrm{R}$. The effect of cigarette smoking and alcohol consumption on efficacy of Helicobacter pylori eradication. Pol Arch Med Wewn 2000;104:569-574.

36. Baena JM, Lopez C, Hidalgo A, et al. Relation between alcohol consumption and the success of Helicobacter pylori eradication therapy using omeprazole, clarithromycin and amoxicillin for 1 week. Eur J Gastroenterol Hepatol 2002;14:291-296.

37. Aydemir S, Boyacioglu S, Gur G, et al. Helicobacter pylori infection in hemodialysis patients: susceptibility to amoxicillin and clarithromycin. World J Gastroenterol 2005;11:842-845.

38. Jung SW, Lee SW, Hyun JJ, et al. Efficacy of Helicobacter pylori eradication therapy in chronic liver disease. Dig Liver Dis 2009;41:134-140.

39. Kim N, Kim JM, Kim CH, et al. Institutional difference of antibiotic resistance of Helicobacter pylori strains in Korea. J Clin Gastroenterol 2006;40:683-687. 